\title{
JORDAN STRUCTURES IN BANACH SPACES
}

\author{
CHO-Ho CHU
}

Abstract. We explain how Jordan algebraic structures in Banach spaces arise from the geometry of symmetric manifolds and discuss some applications.

Mathematics subject classification (2010): 17C65, 32M15, 57C35, 58B20, 46L05, 46L70.

Keywords and phrases: Symmetric domain; Jordan triple systems; Möbius transformation; Bergmann operator; Denjoy-Wolff Theorem.

\section{REFERENCES}

[1] J. ARAZY, Isometries of Banach algebras satisfying the von Neumann inequality, Math. Scand. 74 (1994), 137-151.

[2] É. CARTAN, Sur les domaines bornés homogènes de l'espace de $n$ variables complexes, Abh. Math. Semin. Univ. Hamburg 11 (1935), 116-162.

[3] H. CARTAN, Les fonctions de deux variables complexes et le problème de la représentation analytique, J. Math. Pures et Appl. 10 (1931), 1-114.

[4] C-H. CHU, Jordan structures in geometry and analysis, Cambridge Tracts in Math. 190, Cambridge Univ. Press, Cambridge, 2012.

[5] C-H. CHU, Matrix-valued harmonic functions on groups, J. Reine Angew. Math. 552 (2002), 15-52.

[6] C-H. CHU, Matrix convolution operators on groups, Lecture Notes in Math. 1956, Springer-Verlag, Heidelberg, 2008.

[7] C-H. CHU, Jordan triples and Riemannian symmetric spaces, Advances in Math. 219 (2008), 20292057.

[8] C-H. Chu, H. Hamada, T. Honda And G. Kohr, Distorsion theorems for convex mappings on homogeneous balls, J. Math. Anal. Appl. 369 (2010), 437-442.

[9] C-H. ChU AND P. Mellon, Iteration of compact holomorphic maps on a Hilbert ball, Proc. Amer. Math. Soc. 125 (1997), 1771-1777.

[10] C-H. ChU And P. Mellon, Jordan structures in Banach spaces and symmetric manifolds, Expos. Math. 16 (1998), 157-180.

[11] J. Faraut And A. Koranyi, Analysis on symmetric cones, Clarendon Press, Oxford, 1994.

[12] HARISH-ChandRa, Representations of semi-simple Lie groups VI, Amer. J. Math. 78 (1956), 564 628.

[13] R. IORDĂNESCU, Jordan structures in analysis, geometry and physics, Editura Academiei Române, Bucharest, 2009.

[14] N. JacobSon, Structure and representations of Jordan algebras, Amer. Math. Soc. Colloq. Publ. 39 (1968).

[15] P. Jordan, J. Von Neumann ANd E. Wigner, On an algebraic generalisation of the quantum mechanical formalism, Ann. of Math. 36 (1934), 29-64.

[16] R. V. KADISON, Isometries of operator algebras, Ann. of Math. 54 (1951), 325-338.

[17] I. L. KANTOR, Classification of irreducible transitive differential groups, Dokl. Akad. Nauk SSSR 158 (1964), 1271-1274.

[18] I. L. KAnTOR, Transitive differential groups and invariant connections on homogeneous spaces, Trudy Sem. Vecktor. Tenzor. anal. 13 (1966), 310-398.

[19] W. KaUP, Algebraic characterization of symmetric complex Banach manifolds, Math. Ann. 228 (1977), 39-64. 
[20] W. KAUP, A Riemann mapping theorem for bounded symmetric domains in complex Banach spaces, Math. Z. 183 (1983), 503-529.

[21] W. KAUP AND H. UPMEIER, Banach spaces with biholomorphically equivalent unit balls are isomorphic, Proc. Amer. Math. Soc. 58 (1976), 129-133.

[22] W. KAUP, Hermitian Jordan triple systems and their automorphisms of bounded symmetric domains, in: Non-associative algebra and its applications, Oviedo, 1993 (ed. S. González), Kluwer Acad. Publ. Dordrecht (1994), 204-214.

[23] M. Koecher, Imbedding of Jordan algebras into Lie algebras I, Bull. Amer. J. Math. 89 (1967), 787-816.

[24] M. KOECHER, An elementary approach to bounded symmetric domains, Lecture Notes, Rice University, 1969.

[25] O. Loos, Bounded symmetric domains and Jordan pairs, Mathematical Lectures, University of California, Irvine, 1977.

[26] K. MCCRIMmon, Jordan algebras and their applications, Bull. Amer. Math. Soc. 84 (1978), 612627.

[27] K. MCCRimmon, A taste of Jordan algebras, Universitext, Springer-Verlag, Heidelberg, 2004.

[28] P. Mellon, Holomorphic invariance on bounded symmetric domains, J. Reine Angew. Math. 523 (2000), 199-223.

[29] K. MeyberG, Jordan-Tripelsysteme und die Koecher-Konstruktion von Lie-Algebren, Math. Z. 115 (1970), 58-78.

[30] I. SATAKE, Algebraic strutures of symmetric domains, Princeton Univ. Press, Princeton, 1980.

[31] J. TiTs, Une classe d'algèbres de Lie en relation avec les algèbres de Jordan, Indag. Math. 24 (1962), 530-535.

[32] H. UPMEIER, Über die Automorphismengruppe von Banach-Mannigfaltigkeiten mit invarianter Metrik, Math. Ann. 223 (1976), 279-288.

[33] H. UPMEIER, Jordan algebras in analysis, operator theory and quantum mechanics, CBMS, Amer. Math. Soc., 1987.

[34] J. P. ViguÉ, Le groupe des automorphismes analytiques d'un domaine borné d'un espace de Banach complexe. Application aux domaines bornés symétriques, Ann. Sc. Ec. Norm. Sup. 9 (1976), $203-282$. 